\title{
Pathophysiology and time course of silent myocardial ischaemia during mental stress: clinical, anatomical, and physiological correlates
}

\author{
Suzanne E Legault, Michael R Freeman, Anatoly Langer, Paul W Armstrong
}

\begin{abstract}
Objective-To define the prevalence and pathophysiology of myocardial ischaemia induced by mental stress in patients with coronary artery disease and exercise inducible ischaemia, and to determine the correlation between the severity of coronary artery disease and ischaemia induced by speech.

Design-Prospective cohort study.

Setting-Tertiary care academic institution.

Patients and protocol-47 patients with coronary artery disease and 20 normal controls were studied using standardised exercise and mental stress. The ambulatory nuclear vest provided continuous measures of left ventricular ejection fraction and relative volume changes: an ischaemic response to mental stress was defined as a decrease in ejection fraction of $\geqslant 5 \%$ for $\geqslant 60 \mathrm{~s}$. Severity of coronary artery disease was assessed by the extent of thallium reversibility on exercise testing and the severity of angiographic disease.
\end{abstract}

Results-23 (49\%) of 47 patients with coronary artery disease had an ischaemic response to mental stress which occurred early, was sustained throughout the task and associated with an increase in end systolic volume. In contrast, the pattern of left ventricular response in the remaining 24 patients $(51 \%)$ resembled that in the normal controls. Patients with mental stress induced ischaemia tended to have greater severity of coronary disease (mean (SD) total number of diseased vessels $1.9(0.8)$ v $1.4(0.9), P=$ 0.07 ), more frequent exercise induced angina $(17 / 23 v 7 / 24, P=0.003)$ and lower increases in heart rate (36 (11) $v 49$ (23) beats per $\min , P=0.023$ ) and systolic blood pressure (32 (19) $v 45$ (18) $\mathrm{mm} \mathrm{Hg}$, $P=0.03$ ) during exercise. Left ventricular responses to speech and exercise were compared in the 23 patients with mental stress induced ischaemia: mental stress was associated with a greater decrease in ejection fraction at comparable increases in rate pressure product $(-6.5(6 \cdot 3) \% v$ $4 \cdot 7(11 \cdot 2) \%, P=0 \cdot 0001)$.

Conclusions-These findings suggest that mental stress induction of myocardial ischaemia is common in patients with stable coronary artery disease. Susceptible patients may have more functionally severe coronary disease.
The time course, pattern, and haemodynamic features of mental stress induced ischaemia suggest a dynamic decrease in coronary supply.

(Br Heart f 1995;73:242-249)

Keywords: mental stress; myocardial ischaemia; coronary artery disease; left ventricular function

Several studies have shown that laboratory modelled mental stress is an effective trigger of myocardial ischaemia in patients with coronary artery disease. ${ }^{1-9}$ The prevalence of ischaemic responses varies with the sensitivity of the assessment technique from about $20 \%$ using ST segment depression ${ }^{1-6}$ to $70 \%$ with positron emission tomography of myocardial hypoperfusion. ${ }^{4}$ It is now appreciated that ST depression may be a late and relatively insensitive indicator of myocardial ischaemia. There is compelling evidence that mental stress provokes myocardial ischaemia, with concordance of perfusion defects and wall motion abnormality during mental stress and exercise. ${ }^{4}$ Mental stress induced ischaemia resembles daily life ischaemia ${ }^{10-13}$ in that both are usually asymptomatic and occur at a lower heart rate and blood pressure than exercise induced ischaemia. Despite this evidence of the effectiveness and distinctive mechanism of mental stress induced ischaemia important questions remain:

(a) What is the prevalence of mental stress induced ischaemia in patients with coronary artery disease and exercise inducible ischaemia?

(b) Is there a correlation between the extent of coronary artery disease and the inducibility of ischaemia by mental stress?

(c) Is there a distinctive time course and pattern of left ventricular dysfunction which may elucidate the underlying pathophysiology of mental stress induced ischaemia?

We addressed these questions by utilising a novel nuclear technology, the nuclear vest, to provide continuous accurate assessment of relative left ventricular volume during exercise and mental stress. Our primary objective was to define the prevalence, and delineate the time course and pathophysiology of mental stress induced ischaemia. A second objective was to determine whether conventional indices of the severity of coronary artery disease predict vulnerability to mental stress induced ischaemia. 
Patients and methods PATIENTS

We studied two populations comprising 20 normal controls without a history of cardiac disease, less than two risk factors, normal physical examination and electrocardiogram (ECG), and normal stress ECG; and 47 patients with a clinical diagnosis of coronary artery disease based on symptoms and reversible thallium defects on recent, clinically indicated thallium scintigraphy during Bruce protocol exercise. Patients with coronary artery disease were excluded if they had bundle branch block, unstable angina in the previous 3 months, myocardial infarction in the previous 6 months, or previous coronary bypass surgery.

THALLIUM SCINTIGRAPHY WITH EXERCISE AND CORONARY ANGIOGRAPHY

Thallium scintigraphy data were available in 45 of 47 patients for interpretation by two experienced observers blinded to clinical and angiographic data. Scintigraphy was performed after injection of $2 \mathrm{mCi}$ of thallium201 during maximum symptom limited exercise by Bruce protocol, with planar images obtained at 5 mins and $4 \mathrm{~h}$ after exercise. The anterior, $45^{\circ}$, and $70^{\circ}$ left anterior oblique planar images were acquired for 10 min each and interpreted according to visual scores as previously described by one of us (MRF). ${ }^{14}$ In summary, each planar image was analysed in three segments per view and scored as: 0 , normal; 1 , mild; 2 , moderate; or 3 , severe reduction. A thallium ischaemic score was calculated as the total postexercise score minus the total $4 \mathrm{~h}$ redistribution score. The results of coronary angiography in 40 of 47 patients with coronary artery disease performed for clinical indications within the previous 12 months were interpreted by an experienced angiographer independent from the study. The number of vessels with $\geqslant 50 \%$ stenosis was determined and a jeopardy score as determined by Califf et $a l^{15}$ with maximum score of 9 , was derived.

\section{EXPERIMENTAL PROTOCOL}

Informed consent was obtained from all participants. The protocol was approved by the institutional ethics committee on 23 October 1990. Basic demographic data, cardiac symptoms ratings, and medication status were verified. When possible, antianginal medication was withheld for $48 \mathrm{~h}$ before the study. Table 1 gives the proportion of patients who received antianginal medication during testing. All participants refrained from food, caffeine, and nicotine for $2 \mathrm{~h}$ before the start of testing.

Studies were completed on a single day between 9 am and $1 \mathrm{pm}$. The nuclear VEST was applied, a pre-exercise control obtained, and participants exercised using the protocol of the National Institutes of Health (NIH). After a $15 \mathrm{~min}$ recovery period they were seated in a sound deadened chamber where a series of four mental stress tasks, including three computer-simulated arousal tasks and a personally relevant speech, ${ }^{6}{ }^{16-18}$ was applied.
Each task was preceded by a 10 min control period for stabilisation of haemodynamic parameters; pre-exercise control was standing, whereas premental stress control was sitting. The protocol lasted approximately $4 \mathrm{~h}$, with exercise first and speech last. Because of the higher frequency of abnormal responses during speech, this report is limited to comparison of exercise and speech responses.

Physiological indices measured during control and stress periods included continuous recording of heart rate, frequent measures of blood pressure using an automated cuff, and VEST derived measures of left ventricular ejection fraction, with relative end systolic and end diastolic volumes. An event marker on the VEST was used to permit precise temporal alignment of VEST derived measures with each time point in the task. Participants were questioned about anginal symptoms at the conclusion of each task.

\section{VEST application}

Each participant was injected with red blood cells labelled in vitro with $25 \mathrm{mCi}$ of technetium-99m. Electrocardiographic electrodes recorded an inferior and a modified V5 lead. A multiple gated equilibrium scintigraphic study of the cardiac blood pool was obtained in the $45^{\circ}$ left anterior oblique view and left ventricular ejection fraction was calculated using standard multiple regions of interest and background subtraction as previously described. ${ }^{19}$ The radionuclide detector was positioned over the left ventricle using a gammacamera to determine optimal placement; this was verified by static gammacamera images at the beginning and end of each study. The detector was secured by a plastic garment around the chest wall.

Radionuclide data from the VEST were analysed using a dedicated microcomputer. Cardiac cycles were rejected if the $R R$ interval varied more than $20 \%$ of the average of the previous four cardiac cycles. Ejection fraction was calculated from stroke counts (end diastolic minus end systolic counts divided by fixed background corrected end diastolic counts). The background was determined by the computer to give a baseline ejection fraction similar to that determined with the gated equilibrium study. The electrocardiographic gated radionuclide activity data were summed in $30 \mathrm{~s}$ intervals and trend plots generated. We determined heart rate, ejection fraction, relative end systolic and end diastolic volumes expressed as a per cent of end diastolic volume at the start of the study. The accuracy and reproducibility of ejection fraction measurement have been validated by ourselves ${ }^{20}$ and Yang et al. ${ }^{21}$

\section{Exercise testing}

Participants underwent an upright treadmill exercise test using the protocol of the NIH with 12 lead electrocardiography. It was chosen because the more gradual increase in heart rate and systolic blood pressure provided an opportunity to match heart rate and blood pressure increases during mental stress. 
Exercise end points were moderate chest pain, ST depression $>2 \mathrm{~mm}$, ventricular arrhythmia, fatigue, or attainment of a target heart rate.

\section{Mental stress testing}

Without forewarning, participants were instructed to speak for 3-5 min on their personal faults or undesirable habits to an assembled audience of stern, white coated attendants. Standard instructions were given, as described by Rozanski et al. ${ }^{6}$ Participants were given $4 \mathrm{~min}$ to compose their speech, during which they were alone in the experimental chamber. The audience entered the chamber and the participant started to speak. Haemodynamic measurements were made during the preparation and delivery phases of speech.

DEFINITION OF ISCHAEMIC RESPONSES DURING EXERCISE AND MENTAL STRESS

An ischaemic response to exercise was defined as an increase of $\leqslant 5 \%$ in left ventricular ejection fraction at peak exercise. ${ }^{22}$ An ischaemic response to mental stress was predefined as a decrease of $\geqslant 5 \%$ lasting $\geqslant 60 \mathrm{~s}$ in left ventricular ejection fraction. ${ }^{23}$

\section{DATA PROCESSING AND ANALYSIS}

Baseline values for heart rate, systolic and diastolic blood pressures, left ventricular ejection fraction, and relative end systolic and end diastolic volumes were determined for exercise and mental stress by taking the mean of these measures during the control period preceding each task. Change scores in these physiological indices were derived by subtracting the appropriate control value from the task value: hence change values corrected for differences in control values between tasks and between participants. Change scores were available at $30 \mathrm{~s}$ intervals during exercise and mental stress for VEST derived measures. Change values were available at $150 \mathrm{~s}$ intervals during exercise and $90 \mathrm{~s}$ intervals during mental stress for blood pressure. Data are presented as means (SD) unless otherwise noted.

The SAS statistical package (SAS Institute, Cary, NC, USA) was used for data analysis. Statistical significance was defined as $\mathbf{P}<$ 0.05. Dichotomous variables were compared using $\chi^{2}$ or Fisher's exact test. Continuous variables were compared using the unpaired $t$ test for between participant comparisons and the paired $t$ test for comparisons within participants. Repeated measures analysis of variance (ANOVA) was used to evaluate changes in physiological variables during tasks-that is, exercise and mental stress. A two way repeated measures ANOVA was used for comparison of the time course of change in physiological variables between groups, for example participants with and without speech induced ischaemia. An effect was considered significant only if the overall model had a value of $P<0 \cdot 05$. Multivariate logistic regression modelling of the number of cardiac risk factors, thallium reversibility score, use of $\beta$ blockers, exercise induced angina, heart rate, and blood pressure responses to exercise was performed using the CATMOD procedure in SAS to predict the occurrence of mental stress induced ischaemia.

\section{Results}

\section{BASELINE CHARACTERISTICS}

All normal participants had a mean (SD) age of $42 \cdot 2(7 \cdot 8)$ years and a mean of $1.4(0.5)$ cardiac risk factors. Baseline left ventricular ejection fraction was 58 (10)\%. Participants exercised for a mean of $17.7(2.7)$ min during the NIH protocol, attaining mean increases in heart rate of 73 (14) beats per min, systolic blood pressure of 57 (15) $\mathrm{mm} \mathrm{Hg}$, diastolic blood pressure of 17 (8) $\mathrm{mm} \mathrm{Hg}$, and rate pressure product of 16797 (3301) beats per $\mathrm{min} \times \mathrm{mm} \mathrm{Hg}$. None had electrocardiographic evidence of ischaemia at peak exercise. Ejection fraction rose by $12(10) \%$.

Table 1 gives baseline characteristics, including the thallium reversibility score on Bruce exercise testing, angiographic jeopardy score, and the total number of diseased coronary vessels in the 47 patients with coronary artery disease.

\section{BASELINE HAEMODYNAMIC VALUES BEFORE} EXERCISE AND SPEECH

Pre-exercise baseline data were obtained in the standing position, whereas premental stress baseline data were obtained in the sitting position. Repeated measures ANOVA within baseline periods showed no significant change in haemodynamic variables, indicating a stable physiological environment before task initiation. There were significant differences between pre-exercise and premental stress baseline values for heart rate (patients with coronary artery disease 73 (13) $v 67$ (12) beats per min, $P=0.0001$; normal controls 78 (12) $v 73$ (9) beats per min, $\mathrm{P}=0.001$ ), left ventricular ejection fraction (patients with coronary artery disease $50(8) \% v 46(8) \%$, $\mathrm{P}=0.0001$; normal controls 51 (8)\% $v 45$

Table 1 Baseline characteristics of 47 patients with coronary artery disease

\begin{tabular}{lc}
\hline Mean (SD) age (years) & $54 \cdot 5(7 \cdot 0)$ \\
Mean (SD) years since diagnosis of coronary & \\
artery disease & $3 \cdot 2(4 \cdot 6)$ \\
Mean (SD) total no of risk factors for coronary & \\
artery disease & $2 \cdot 6(0 \cdot 9)$ \\
Family history of coronary artery disease (\%) & $36(77)$ \\
Smoking (\%) & $24(51)$ \\
High cholesterol concentration (\%) & $26(55)$ \\
Diabetes mellitus (\%) & $20(43)$ \\
Hypertension (\%) & $17(36)$ \\
CCS symptom class (\%): & $36(77)$ \\
Class 1 & $8(17)$ \\
Class 2 & $3(6)$ \\
Class 3 & $41(87)$ \\
Angina with exertion (\%) & $23(49)$ \\
Angina with emotional upset (\%) & $17(36)$ \\
Previous myocardial infarction (\%) & \\
Current cardiac medication: & $21(45)$ \\
Nitrates (\%) & $9(19)$ \\
$\beta$ Blockers (\%) & $17(36)$ \\
Calcium antagonists (\%) & $31(66)$ \\
Acetylsalicylic acid (\%) & $5 \cdot 2(3 \cdot 3)$ \\
Mean (SD) total thallium reversibility score & $3 \cdot 9(2 \cdot 2)$ \\
(Bruce exercise test) & $1 \cdot 7(0 \cdot 9)$ \\
Mean (SD) jeopardy score & \\
Mean (SD) total no of diseased vessels & \\
$\quad$ (coronary stenosis $\geqslant 50 \%)$ & \\
\hline
\end{tabular}

CCS, Canadian Cardiovascular Society. 
(7) $\%, P=0.0002)$, and left ventricular end diastolic volume (patients with coronary artery disease $92(9) \%$ v $84(10) \%, P=$ 0.0001; normal controls 93 (8)\% v 84 (9)\%, $\mathbf{P}=0.0002)$. These differences are presumed to reflect effects of posture and decay in counts over the time course of the protocol.

\section{SPEECH INDUCED ISCHAEMIA: PREVALENCE}

AND CORRELATES

Patients with coronary artery disease were divided, as prospectively defined, into two

Table 2 Baseline characteristics according to ischaemia induced by speech

\begin{tabular}{|c|c|c|c|}
\hline & $\begin{array}{l}\text { Patients without } \\
\text { speech induced } \\
\text { ischaemia } \\
(n=24)\end{array}$ & $\begin{array}{l}\text { Patients with } \\
\text { speech induced } \\
\text { ischaemia } \\
(n=23)\end{array}$ & p value \\
\hline \multirow{2}{*}{$\begin{array}{l}\text { Mean (SD) age (years) } \\
\text { Mean (SD) years since diagnosis of coronary } \\
\text { artery disease }\end{array}$} & $53 \cdot 0(6 \cdot 6)$ & $56 \cdot 1(7 \cdot 2)$ & $0 \cdot 1$ \\
\hline & $2.3(2.3)$ & $4 \cdot 2(6 \cdot 1)$ & $0 \cdot 2$ \\
\hline \multirow{2}{*}{\multicolumn{4}{|c|}{ Mean (SD) total no of risk factors for coronary }} \\
\hline & $2 \cdot 4(1 \cdot 0)$ & $2.9(0.7)$ & 0.06 \\
\hline Family history of coronary artery disease (\%) & $18(75)$ & $18(78)$ & $1 \cdot 0$ \\
\hline Smoking (\%) & $10(42)$ & $14(61)$ & $0 \cdot 2$ \\
\hline High cholesterol concentration (\%) & $12(50)$ & $14(61)$ & 0.6 \\
\hline Diabetes mellitus (\%) & $9(38)$ & $11(48)$ & $0 \cdot 6$ \\
\hline Hypertension (\%) & $8(33)$ & $9(39)$ & $0 \cdot 8$ \\
\hline \multicolumn{4}{|l|}{ CCS symptom class (\%): } \\
\hline Class 1 & $20(83)$ & $16(70)$ & \\
\hline Class 2 & $4(17)$ & $4(17)$ & \\
\hline Class 3 & $0(0)$ & $3(13)$ & \\
\hline Angina with exertion (\%) & $21(88)$ & $20(87)$ & $1 \cdot 0$ \\
\hline Angina with emotional upset (\%) & $12(50)$ & $11(48)$ & $1 \cdot 0$ \\
\hline Previous myocardial infarction (\%) & $10(42)$ & $7(30)$ & $0 \cdot 5$ \\
\hline \multicolumn{4}{|l|}{ Cardiac medication: } \\
\hline Nitrates (\%) & $11(46)$ & $10(43)$ & $0 \cdot 7$ \\
\hline$\beta$ Blockers $(\%)$ & $7(29)$ & $2(9)$ & $0 \cdot 14$ \\
\hline Calcium antagonists (\%) & $9(38)$ & $8(35)$ & $0 \cdot 7$ \\
\hline Acetylsalicylic acid (\%) & $18(75)$ & $13(56)$ & $0 \cdot 2$ \\
\hline \multirow{5}{*}{$\begin{array}{l}\text { Mean (SD) thallium reversibility score } \\
\text { (Bruce exercise test) } \\
\text { Mean (SD) jeopardy score } \\
\text { Mean (SD) total no of diseased vessels } \\
\text { (coronary stenosis } \geqslant 50 \% \text { ) }\end{array}$} & & & \\
\hline & $4 \cdot 3(2 \cdot 9)$ & $6 \cdot 0(3 \cdot 6)$ & 0.08 \\
\hline & $3 \cdot 4(2 \cdot 2)$ & $4 \cdot 3(2 \cdot 3)$ & $0 \cdot 2$ \\
\hline & & & \\
\hline & $1.4(0.9)$ & $1.9(0.8)$ & 0.07 \\
\hline
\end{tabular}

CCS, Canadian Cardiovascular Society.

Table 3 Response to NIH exercise testing according to ischaemia induced by speech

\begin{tabular}{|c|c|c|c|}
\hline & $\begin{array}{l}\text { Patients without } \\
\text { speech induced } \\
\text { ischaemia } \\
(n=24)\end{array}$ & $\begin{array}{l}\text { Patients with } \\
\text { speech induced } \\
\text { ischaemia } \\
(n=23)\end{array}$ & Pvalue \\
\hline Mean (SD) exercise duration & $12 \cdot 4(4 \cdot 8)$ & $10.9(4 \cdot 4)$ & $0 \cdot 3$ \\
\hline Termination due to angina (\%) & $7(29)$ & $17(74)$ & 0.003 \\
\hline ST depression $\geqslant 1 \mathrm{~mm}(\%)$ & $8(33)$ & $11(48)$ & $0 \cdot 4$ \\
\hline Ischaemic response $(\%)^{\star}$ & $10(42)$ & $14(61)$ & 0.25 \\
\hline $\begin{array}{l}\text { Mean (SD) peak heart rate } \\
\text { (beats per minute) } \dagger\end{array}$ & $49(23)$ & $36(11)$ & 0.023 \\
\hline $\begin{array}{l}\text { Mean (SD) peak systolic blood pressure } \\
(\mathrm{mm} \mathrm{Hg}) \dagger\end{array}$ & $45(18)$ & $32(19)$ & 0.03 \\
\hline $\begin{array}{l}\text { Mean (SD) peak diastolic blood pressure } \\
(\mathrm{mm} \mathrm{Hg}) \dagger\end{array}$ & $14(8)$ & $14(8)$ & 0.94 \\
\hline $\begin{array}{l}\text { Mean (SD) peak-rate pressure product } \\
\text { (beats per minute } \times \mathrm{mm} \mathrm{Hg}) \dagger\end{array}$ & $11489(4827)$ & $8664(3681)$ & 0.03 \\
\hline Mean (SD̄) peak ejection fraction (\%)† & $6(9)$ & $2(11)$ & $0 \cdot 16$ \\
\hline Mean (SD) peak end systolic volume (\%) $\dagger$ & $4(12)$ & $7(12)$ & 0.4 \\
\hline Mean (SD) peak end diastolic volume $(\%) \dagger$ & $22(12)$ & $17(7)$ & $0 \cdot 15$ \\
\hline
\end{tabular}

${ }^{\star}$ Defined as an increase of $\leqslant 5 \%$ in left ventricular ejection fraction at peak exercise. + Change from baseline value.

Table 4 Severity of coronary artery disease according to ejection fraction response to NIH exercise testing

\begin{tabular}{|c|c|c|c|}
\hline & $\begin{array}{l}\text { Increase in left } \\
\text { ventricular ejection } \\
\text { fraction } \geqslant 5 \% \\
(n=24)\end{array}$ & $\begin{array}{l}\text { Increase in left } \\
\text { ventricular ejection } \\
\text { fraction }<5 \% \\
(n=23)\end{array}$ & $P$ value \\
\hline $\begin{array}{l}\text { Mean (SD) thallium reversibility score } \\
\text { (Bruce exercise test) } \\
\text { Mean (SD) jeopardy score } \\
\text { Mean (SD) total no of diseased vessels } \\
\text { (coronary stenosis } \geqslant 50 \% \text { ) }\end{array}$ & $\begin{array}{l}4 \cdot 6(3 \cdot 4) \\
3 \cdot 6(2 \cdot 3)\end{array}$ & $\begin{array}{l}5 \cdot 7(3 \cdot 3) \\
4 \cdot 1(2 \cdot 3)\end{array}$ & $\begin{array}{l}0.3 \\
0.5\end{array}$ \\
\hline
\end{tabular}

groups based on the development of an ischaemic response during speech: $23(49 \%)$ had an ischaemic response, whereas 24 (51\%) had a non-ischaemic response. Mean (SD) peak changes in left ventricular ejection fraction were $-8.0(6.7) \%$ in patients with mental stress induced ischaemia and $3.5(6.8) \%$ in those without an ischaemic response to speech $(t=5 \cdot 8, \mathrm{P}=0.0001)$. The two groups had similar increases in heart rate and blood pressure; moreover, when changes in heart rate, and systolic and diastolic blood pressures during speech were correlated with the peak change in left ventricular ejection fraction there was no association in the sample of 47 patients or within the subgroup with mental stress induced ischaemia. Two patients with speech induced ischaemia and one without an ischaemic response to speech reported angina during speech; all had an ischaemic left ventricular response to the exercise protocol of the NIH.

Table 2 compares baseline demographic and cardiovascular disease characteristics of patients with and without an ischaemic response to speech. Patients with speech induced ischaemia seemed to have more severe coronary disease, as evidenced by trends for a greater number of cardiac risk factors, a higher thallium reversibility score, a higher angiographic jeopardy score, and a greater mean number of diseased coronary vessels. However, these differences were not statistically significant.

A greater proportion of patients without an ischaemic response to speech was taking $\beta$ blockers $(7 / 24 v 2 / 23, P=0 \cdot 1)$. Furthermore, two way ANOVA for prediction of speech induced ischaemia showed a significant interaction between $\beta$ blockers and angiographic evidence of disease severity. In the subgroup not taking $\beta$ blockers $(n=38)$, patients with speech induced ischaemia had a significantly higher jeopardy score $(4 \cdot 7(2 \cdot 2)$ v $2 \cdot 8(1 \cdot 7), \mathrm{P}$ $=0.01$ ) and a greater number of diseased vessels $(2 \cdot 1(0.8)$ v $1.2(0.8), \mathrm{P}=0.008)$ compared with those without an ischaemic response to speech. There were no significant differences in angiographic disease severity in the subgroup $(n=9)$ taking $\beta$ blockers.

Table 3 gives responses to the exercise protocol of the NIH in patients with and without speech induced ischaemia. Patients with speech induced ischaemia more often terminated exercise because of angina, attained lesser increases in heart rate and systolic blood pressure at peak exercise, but had similar left ventricular responses to those of patients without an ischaemic response to speech. A multivariate model using previously described indices of the severity of coronary disease did not identify any index or combination of indices that were significant for predicting speech induced ischaemia.

Some 24 patients (51\%) had an ischaemic response defined as an increase of $\leqslant 5 \%$ in left ventricular ejection fraction at peak exercise to the exercise protocol of the $\mathrm{NIH}$, which did not correlate with speech induced ischaemia (table 4). In contrast to 
speech induced ischaemia, exercise induced ischaemia was not associated with angiographic evidence of disease severity or thallium reversibility score (table 4).

\section{PATHOPHYSIOLOGY OF SPEECH INDUCED} ISCHAEMIA

Figure 1 shows the time course of change in rate pressure product, ejection fraction, and end systolic and end diastolic volumes during speech in 19 normal controls (one refused to participate). There was an initial small increase in rate pressure product during the

Figure 1 Response to speech in 19 normal controls. Data points are mean (SEM).
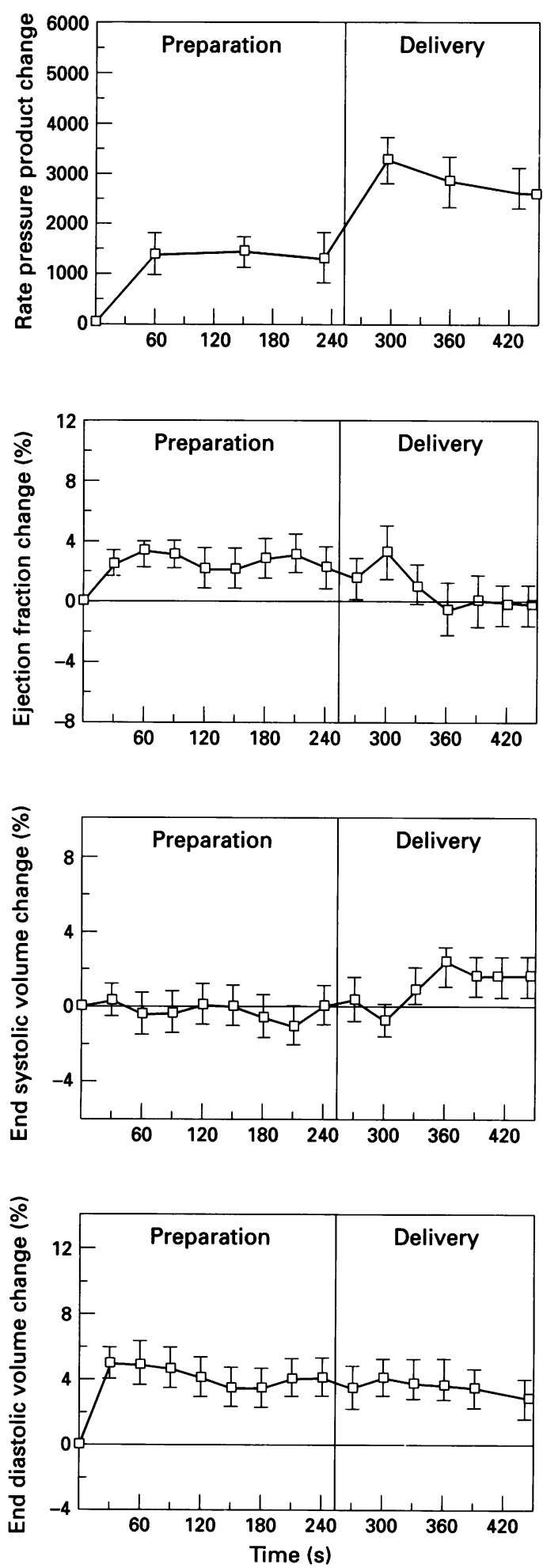

preparation phase, which was accompanied by a rise in ejection fraction and end diastolic volume. Rate pressure product increased substantially at the onset of speech delivery and the ejection fraction was initially maintained. As speech delivery proceeded, the ejection fraction decreased to a value corresponding to that of baseline; this change seemed to be mediated through a mild rise in end systolic volume. The mean peak change in heart rate was $9.6(11.9)$ beats per min, systolic blood pressure $20.2(13.5) \mathrm{mm} \mathrm{Hg}$, diastolic blood pressure $12.6(8.1) \mathrm{mm} \mathrm{Hg}$, left ventricular ejection fraction $-0.6(1.6) \%$, end systolic volume $2.5(0.9) \%$, and end diastolic volume $3 \cdot 7(1 \cdot 4) \%$.

Figure 2 shows the time course of change in rate pressure product, ejection fraction, and end systolic and end diastolic volumes in patients with and without an ischaemic response to speech. Patients with speech induced ischaemia showed a significantly different pattern of ejection fraction response throughout the speech task compared with that of those without an ischaemic response; an initial decrease during preparation was followed by a more pronounced decrease during delivery (group effect $P=0.0001$, time effect $P=0.0001)$. This was associated with a greater increase in end systolic volume, which was also accentuated at the start of speech delivery (group effect $P=0.0001$, time effect $P=0.0001)$. End diastolic volume increased significantly more in the patients without an ischaemic response to speech, but the temporal pattern was not different (group effect $P=$ 0.004 , time effect $P=0 \cdot 11$ ). Differences between patients with and without an ischaemic response in ejection fraction, and end systolic and end diastolic volumes were already significant during the preparation phase of speech $(P=0.0002, P=0.0007$ and $P=0.03$, respectively). The magnitude, pattern, and time course of heart rate, systolic and diastolic blood pressure, and rate pressure product increase during speech was identical in patients with and without an ischaemic response to speech. There were no differences in heart rate, blood pressure, or left ventricular responses with respect to $\beta$ blocker medication.

Left ventricular responses to speech and exercise in the 23 patients with mental stress induced ischaemia were compared at matched levels of rate pressure product to examine further the pathophysiology of mental stress induced ischaemia. Rate pressure product was comparable between stage 1 exercise and at the 2.5 min mark of speech delivery. At comparable rate pressure products, speech was associated with a substantial decrease in left ventricular ejection fraction compared with a mild increase with exercise $(-6 \cdot 5(6 \cdot 3) \% v$ $4 \cdot 7(11 \cdot 2) \%, P=0.0001$ (fig 3). Whereas exercise was associated with a substantially greater increase in end diastolic volume $(16.4$ $(9.2) \% v 1.6(5.5) \%, P=0.0001)$, speech was associated with a greater rise in end systolic volume $(6.7(6.3) \%$ v $2.9(11.3) \%, P=0.05)$ (fig 3). 
Figure 2 Response to speech in 47 patients with coronary artery disease. Response to speech in 23 patients with ischaemic responses during speech (-) and 24 patients with non-ischaemic responses during the same task (mean $(S E M) .{ }^{*} P<0.01$ patients with speech induced ischaemia versus those without speech induced ischaemia, two way ANOVA. $\star * P<$ 0.001 , change over time, two way ANOVA.

\section{Discussion}

In this study, continuous assessment of left ventricular volume with the ambulatory nuclear VEST provided new insight into the pathophysiology of myocardial ischaemia during mental stress. Our data show that the ischaemic response to personally relevant speech was sudden, starting in the preparation phase and accentuated during speech delivery. The decrease in ejection fraction was associated with an increase in end systolic volume suggesting myocardial ischaemia. In contrast, patients with a non-ischaemic response
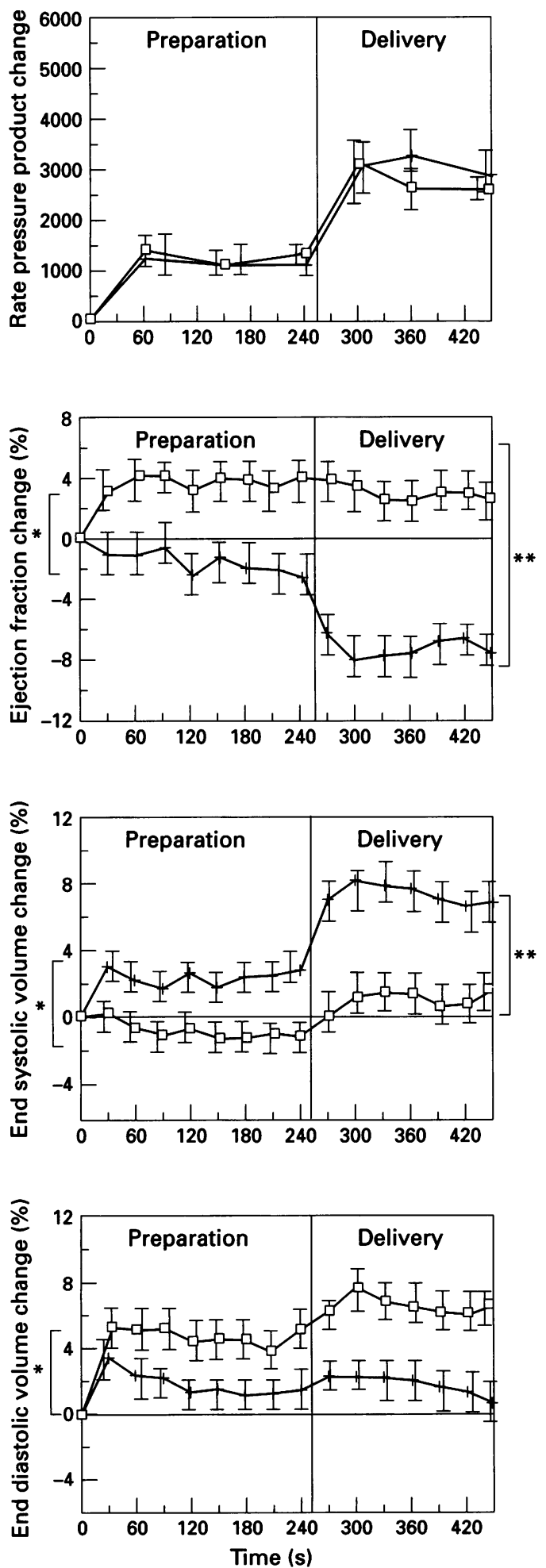
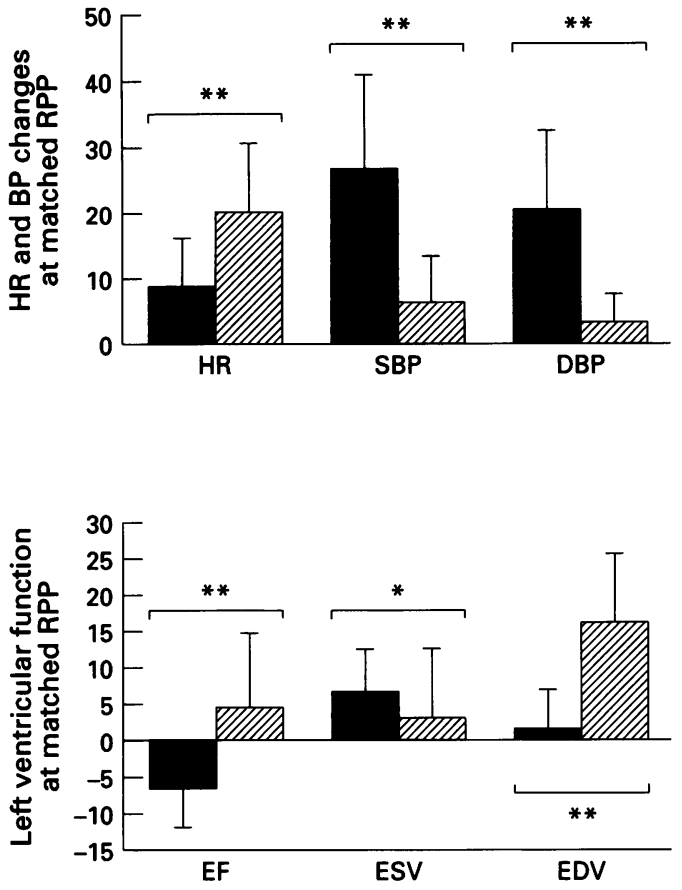

Figure 3 Cardiovascular responses to speech ( $\square$ ) and exercise (10) compared at matched levels of rate pressure product (RPP) in 23 patients with ischaemia induced by mental stress. $R P P$ was increased to a similar extent during stage 1 NIH exercise and 2.5 min into speech delivery (3354 (1813) v 3268 (2186) beats per $\min \times \mathrm{mm} \mathrm{Hg}$ respectively, $P=0.9)$. Changes in $(A)$ heart rate $(H R)$ (beats per min) systolic blood pressure (SBP), and diastolic blood pressure (DBP) (mm $\mathrm{Hg}$ ); and (B) ejection fraction (EF) end systolic volume (ESV), and end diastolic volume (EDV) during speech and exercise. ${ }^{\star} P<0.05$, paired $t$ test. ${ }^{\star \star P} P<0.001$, paired $t$ test.

resembled normal controls by showing maintenance of, or slight increase in, ejection fraction, with accompanying increase in end diastolic volume but no change in end systolic volume.

Provocation of ischaemia during mental stress was not attributable to a greater increase in myocardial oxygen demand in patients with speech induced ischaemia. Rather patients with and without an ischaemic response to speech had similar increases in heart rate and blood pressure; moreover, patients with speech induced ischaemia had lesser increases in end diastolic volume during speech than those without an ischaemic response to speech. These data suggest that patients with speech induced ischaemia had a relative reduction in myocardial oxygen supply during mental stress, which could be caused by either greater fixed coronary stenoses or greater dynamic reduction in coronary blood flow. Our data show that patients with speech induced ischaemia had more severe fixed coronary artery stenoses; this trend became significant when the confounding effect of $\beta$ blocker medication was removed.

Moreover, by performing a within subject comparison of physiological responses to mental stress and exercise in the 23 patients with speech induced ischaemia (fig 3 ), we have strong evidence that a dynamic reduction in coronary blood flow is important in the 
development of ischaemia during mental stress-that is, at a matched level of myocardial oxygen demand between the two tasks, mental stress was associated with myocardial ischaemia (as evidenced by a decrease in ejection fraction), whereas the exercise response was non-ischaemic (as evidenced by an increase in ejection fraction). As this within subject comparison effectively matches conditions between exercise and mental stress for two of the three potential causes of ischaemia-that is, severity of fixed coronary disease and increase in myocardial oxygen demand, the only factor accounting for mental stress induced ischaemia is a dynamic reduction in coronary blood flow. In keeping with our data, Yeung et al ${ }^{24}$ reported direct angiographic visualization of coronary vasoconstriction during mental stress, supporting the concept of reduced coronary flow as a significant pathophysiological mechanism of mental stress induced ischaemia. Furthermore, Yeung et $a l^{24}$ found that mental stress induced coronary vasoconstriction occurred only at sites with atherosclerotic plaque; one could postulate that more extensive coronary disease would lead to more opportunity for vasoconstriction and therefore a greater likelihood of myocardial ischaemia during mental stress.

Of note, our matching of the level of increase in rate pressure product between tasks may not represent accurate estimates of myocardial oxygen demand in the two circumstances. As can be seen from fig 3, the increase in rate pressure product was determined by different factors in exercise versus speech. An additional determinant of myocardial oxygen demand not matched in this comparison is end systolic volume, with much greater increases in this index during speech. Consideration of the time course of left ventricular volume change during speech (fig 2), however, would suggest that the greater rise in end systolic volume accompanies rather than causes the decrease in ejection fraction during speech.

Although indices of disease severity tended to be greater in patients with mental stress induced ischaemia, a multivariate model did not predict mental stress induced ischaemia. This may reflect either low statistical power due to the limited sample size, or the difference in pathophysiology of ischaemia during exercise versus mental stress in patients with coronary artery disease.

Although the study was designed to test patients not taking antianginal medication, this was not always possible. A subsidiary analysis was planned to examine the potential protective effect of $\beta$ blockers, in particular against mental stress induced ischaemia. ${ }^{25} \mathrm{We}$ found that treatment with $\beta$ blockers may have protected against mental stress induced ischaemia given the observed trends for more patients without an ischaemic response to speech to be taking $\beta$ blockers and those with speech induced ischaemia who were not taking $\beta$ blockers to have significantly greater angiographic evidence of disease severity.
Such a protective effect may be mediated by an increase in coronary reserve during mental stress, analogous to the observations by Bortone et $a l^{26}$ on the effect of $\beta$ blockers to increase coronary reserve during exercise testing in patients with coronary artery disease. Bairey et $a l^{25}$ also provided data suggesting that $\beta$ blockers may protect against mental stress induced ischaemia.

Prevalence of mental stress induced ischaemia in our patients with coronary artery disease and thallium reversibility during standard exercise testing was $48 \%$. This value accords with that of previous studies which have used sensitive measures to assess myocardial ischaemia during mental stress. ${ }^{46}$ It is noteworthy that, despite all our patients having thallium reversibility on recent Bruce exercise testing, the sample had relatively mild coronary disease, as evidenced by their Canadian Cardiovascular Society functional class, infrequent ST depression during NIH exercise, and angiographic jeopardy score.

In this study, an ischaemic response to exercise (defined as either an ischaemic left ventricular ejection fraction response or ST depression $\geqslant 1 \mathrm{~mm}$ ) did not correlate with indices of severity of coronary artery disease. This lack of correlation is unexpected and may be the result of the fact that the patient sample had moderate disease severity.

In conclusion, these findings suggest that vulnerability to mental stress induced ischaemia is related to more extensive angiographic disease and that the mechanism of this association is through a dynamic reduction in coronary flow at the site of atherosclerotic plaques.

\section{LIMITATIONS}

Our study used a measure of global ventricular function - that is, left ventricular ejection fraction to assess ischaemia during mental stress. It could be argued that the ejection fraction decrease of $5 \%$ during mental stress may not represent ischaemia but may be secondary to changes in afterload. However, we showed that identical changes in heart rate and blood pressure were observed in patients with or without a decrease in ejection fraction during mental stress. In addition, the magnitude of heart rate and blood pressure change did not correlate with the magnitude of ejection fraction change in either the study sample or the subgroup who developed ischaemia during speech. Hence, it is unlikely that the reduction in ejection fraction is explained by changes in afterload.

There were differences in left ventricular ejection fraction and end diastolic volume between exercise and speech controls. The higher control value of ejection fraction before exercise is likely to be related to upright posture and anticipation of exercise. The lower end diastolic volume before speech is related to decay in counts, and gradual loss of the ${ }^{99 \mathrm{~m}} \mathrm{Tc}$ label to red blood cells over the time course of the protocol, as well as a reduction in blood volume after exercise. The use of change values to compare responses to 
exercise and speech tasks corrected for these small differences in control values.

\section{IMPLICATIONS}

Our findings suggest that mental stress induced ischaemia is a common phenomenon in patients with coronary artery disease and exercise inducible ischaemia. Patients with a normal exercise response on ECG may have ischaemic responses to mental stress.

It is unknown whether vulnerability to mental stress induced ischaemia has prognostic significance or predicts silent ischaemia during daily life. Given the evidence that mental stress induced ischaemia is mediated mainly through coronary vasoconstriction, mental stress testing may provide data on the functional severity of coronary disease which is complementary to that of standard exercise testing.

We wish to acknowledge our research nurse Halina Nawrocki, the technical assistance provided by Dr Ling de Yang and Terry Hsia and database management provided by Lois Adams. This work was supported by the Heart and Stroke Foundation of Ontario.

1 Schiffer F, Hartley LH, Schulman CL, et al. The quiz electrocardiogram: a new diagnostic and research technique trocardiogram: a new diagnostic and research technique for evaluating the relation between emotional stress
ischaemic heart disease. Am $₹$ Cardiol 1976;37:41-7.

2 Specchia G, de Servi S, Falcone C, et al. Mental arithmetic stress testing in patients with coronary artery disease. stress testing in patients with
$A m$ Heart $\mathcal{f} 1984 ; 108: 56-63$

3 Jennings JR, Follansbee WP. Task-induced ST segment depression, ectopic beats, and autonomic responses in coronary heart disease patients. Psychosom Med 1985;47:415-30.

4 Deanfield JE, Kensett M, Wilson RA, et al. Silent myocardial ischaemia due to mental stress. Lancet 1984;ii: 1001-4.

5 Modena MG, Corghi F, Fantini G, et al. Echocardiographic monitoring of mental stress test in ischemic heart disease. Clin Cardiol 1989;12:21-4.

6 Rozanski A, Bairey CN, Krantz DS, et al. Mental stress and the induction of silent myocardial ischaemia in patients with coronary artery disease. $N$ Engl $f \mathrm{Med}$ patients with coro

7 LaVeau PJ, Rozanski A, Krantz DS, et al. Transient left ventricular dysfunction during provocative mental stress in patients with
8 Breisblatt WM, Weiland FL, McLain JR, et al. Usefulness of ambulatory radionuclide monitoring of left ventricular function early after myocardial infarction for predicting residual myocardial ischemia. Am $f$ Cardiol 1988;62: 1005-10.

9 Ironson G, Barr Taylor C, Boltwood M, et al. Effects of anger on left ventricular ejection fraction in coronary artery disease. Am $\mathcal{F}$ Cardiol 1992;70:281-5.

10 Deanfield JE, Shea MJ, Selwyn AP. Clinical evaluation of transient myocardial ischemia during daily life. $A m \mathfrak{F}$ Med 1985;79:18-24.

11 Deanfield JE, Shea MJ, Ribiero P, et al. Transient ST-segment depression as a marker of myocardial ischemia during daily life. Am $\mathcal{F}$ Cardiol 1984;54:1 195-1200.

12 Deanfield JE, Selwyn AP, Chierchia S, et al. Myocardial ischaemia during daily life in patients with stable angina: its relation to symptoms and heart rate changes. Lancet its relation to s

13 Cohn PF. Silent myocardial ischemia: classification, prevalence, and prognosis. $\mathrm{Am} \mathcal{F} \mathrm{Med}$ 1985;79:2-6.

14 Hendler AL, Greyson ND, Robinson MG, Freeman MR. Patients with symptomatic ischaemia have larger thallium perfusion abnormalities and more adverse prognosis than patients with silent ischemia. Can $₹$ Cardiol 1992; 8:814-8.

15 Califf RM, Phillips HR, Hindman MC, et al. Prognostic value of a coronary jeopardy score. $\mathcal{F} \mathrm{Am}$ Coll Cardiol 1985;5:1055-63.

16 Jennings JR. Beat-by-beat vascular responses during anticipatory heart rate deceleration. Physiol Psychol 1982;10:422-30.

17 Saab P, Matthews KA, Stoney CM, McDonald RM. Premenopausal and postmenopausal women differ in Premenopausal and postmenopausal women differ in their cardiovascular and neuroendocrine responses to
behavioral stressors. Psychophysiology 1989;26:270-80.

18 Stirling N. Stroop interference: an input and an output phenomenon. $Q \mathcal{F}$ Exp Psychol 1979;31:121-32.

19 Armstrong PW, Baigrie RS, Daly PA, et al. Tissue plasminogen activator: Toronto (TPAT) placebo-controlled randomized trial in acute myocardial infarction. $\mathcal{F} \mathrm{Am}$ Coll Cardiol 1989;13:1469-76.

20 Freeman MR, Yang L, Hsia T, Adams L, Armstrong PW. Validation of the ambulatory nuclear VEST for accurate assessment of left ventricular ejection fraction during upright bicycle exercise [abstract]. Circulation 1990;82 (suppl III): 136 .

21 Yang L, Bairey CN, Berman DS, et al. Accuracy and reproducibility of left ventricular ejection fraction measurement using an ambulatory radionuclide left ventricular function monitor. $₹$ Nucl Med 1991;32:796-802.

22 Borer JS, Kent KM, Bacharach SL, et al. Sensitivity, speciBorer JS, Kent KM, Bacharach SL, et al. Sensitivity, speci-
ficity and predictive accuracy of radionuclide cineangiography during exercise in patients with coronary artery disease: comparison with exercise electrocardiography. Circulation 1979;60:572-80.

23 Bairey CN, Yang L, Berman DS, et al. Comparison of physiologic ejection fraction responses to activity of daily living: implication for clinical testing. $\mathcal{F} \mathrm{Am}$ Coll Cardiol 1990;16:847-54.

24 Yeung AC, Vekshtein VI, Krantz DS, et al. The effect of atherosclerosis on the vasomotor response of coronary arteries to mental stress. $N$ Engl $f$ Med 1991;325: 1551-6.

25 Bairey CN, Krantz DS, DeQuattro V, Berman DS, Rozanski A. Effect of beta-blockade on low heart raterelated ischemia during mental stress. $\mathcal{F} \mathrm{Am}$ Coll Cardiol 1991;17:1388-95.

26 Bortone AS, Hess OM, Gaglione A, et al. Effect of intravenous propranolol on coronary vasomotion at rest and
during dynamic exercise in patients with coronary artery disease. Circulation 1990;81:1225-35. 